\title{
Event-Related Phase Synchronization Propagates Rapidly across Human Ventral Visual Cortex
}

\author{
Oscar Woolnough ${ }^{1,2}$, Kiefer J. Forseth ${ }^{1,2}$, Patrick S. Rollo ${ }^{1,2}$, Zachary J. Roccaforte ${ }^{1,2}$, Nitin Tandon ${ }^{1,2,3,{ }^{*}}$ \\ ${ }^{1}$ Vivian L. Smith Department of Neurosurgery, McGovern Medical School at UT Health Houston, \\ Houston, TX, 77030, United States of America \\ ${ }^{2}$ Texas Institute for Restorative Neurotechnologies, University of Texas Health Science Center at \\ Houston, Houston, TX, 77030, United States of America \\ ${ }^{3}$ Memorial Hermann Hospital, Texas Medical Center, Houston, TX, 77030, United States of America \\ * Correspondence: nitin.tandon@uth.tmc.edu
}

\section{Abstract}

2 Visual inputs to early visual cortex integrate with semantic, linguistic and memory inputs in higher visual cortex, in a manner that is rapid and accurate, and enables complex computations such as face recognition and word reading. This implies the existence of fundamental organizational principles that enable such efficiency. To elaborate on this, we performed intracranial recordings in 82 individuals while they performed tasks of varying visual and cognitive complexity. We discovered that visual inputs induce highly organized posterior-to-anterior propagating patterns of phase modulation across the ventral occipitotemporal cortex. At individual electrodes there was a stereotyped temporal pattern of phase progression following both stimulus onset and offset, consistent across

10 trials and tasks. The phase of low frequency activity in anterior regions was predicted by the prior 11 phase in posterior cortical regions. This spatiotemporal propagation of phase likely serves as a feed12 forward organizational influence enabling the integration of information across the ventral visual 13 stream. This phase modulation manifests as the early components of the event related potential; 14 one of the most commonly used measures in human electrophysiology. These findings illuminate 15 fundamental organizational principles of the higher order visual system that enable the rapid 16 recognition and characterization of a variety of inputs. 


\section{Introduction}

18 Ventral occipitotemporal cortex is organized in a cortical hierarchy from early visual regions (e.g. calcarine cortex) to associative areas (e.g. fusiform cortex) (Felleman and Van Essen, 1991; Morán et al., 1987), that constitutes the ventral visual stream (Mishkin et al., 1983). This processing pathway is crucial for the integration of visual processing with language and memory (Forseth et al., 2018; Ghuman et al., 2014; Hirshorn et al., 2016; Kadipasaoglu et al., 2016; Tang et al., 2014; Woolnough et al., 2021b), but it is unclear how the propagation of information between functional components of the human ventral visual pathway is coordinated.

Recently, spatial propagation of information has been characterized, in both humans and non-human primates, as travelling waves of cortical activation. These occur at both the macro and micro scale and represent the structured propagation of information across the cortical surface in response to inputs (Lozano-Soldevilla and VanRullen, 2019; Muller et al., 2018, 2014; Sato et al., 2012) or may occur spontaneously (Bahramisharif et al., 2013; Davis et al., 2020; Halgren et al., 2019; Muller et al., 2016; Zhang et al., 2018). In the ventral visual stream of macaques this manifests as a rapid feedforward sweep of activation following visual stimulation (Lamme and Roelfsema, 2000). This spatiotemporal organization, manifest as a travelling wave indexes synchronized long-range corticocortical connections, enabling the integration of information across multiple cortical areas (Sato et al., 2012; Zhang et al., 2018) and perhaps also facilitates predictive coding (Alamia and VanRullen, 2019; Arnal and Giraud, 2012). Such travelling waves have commonly been characterized as a spatial propagation of phase. Sensory inputs result in the modulation of phase of ongoing oscillations (Howard and Poeppel, 2012; Lakatos et al., 2008, 2005; Luo et al., 2010) and the timing of inputs relative to the phase of ongoing oscillations impacts perception (Bonnefond and Jensen, 2012; Davis et al., 2020; Dugué et al., 2015; Forseth et al., 2020; Mercier et al., 2015). Based on this, we hypothesize there is a stimulus-evoked modulation of phase that spatiotemporally propagates through the human ventral visual pathway.

To probe the spread of visually-evoked phase modulation across the ventral visual stream, we utilized high spatiotemporal resolution intracranial recordings in a large human cohort (82 patients), with almost ubiquitous coverage of the ventral visual stream (1,929 electrodes), who performed a language task with relatively low visual complexity stimulus (word reading) or a memory task with a high complexity visual stimulus (face naming). 


\section{Results}

4882 patients semi-chronically implanted with subdural grid electrodes (SDEs; 14 patients) or

49 stereotactically placed depth electrodes (sEEGs; 68 patients) for the localization of intractable 50 epilepsy performed word reading $(n=40)$ and face naming $(n=57)$ tasks (Figure $1 A, B)$. Phase 51 modulation was quantified by computing the inter-trial phase coherence (ITC; Figure 1E,F) of the 52 broadband low frequency signal $(2-30 \mathrm{~Hz})$ at each electrode, across multiple trials.

A

A

$1.5 \mathrm{~s}$

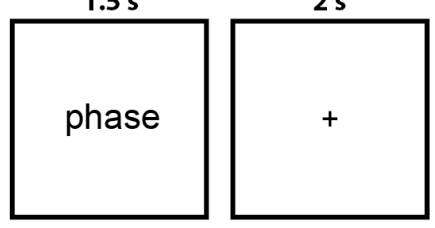

C

$\mathrm{R}$
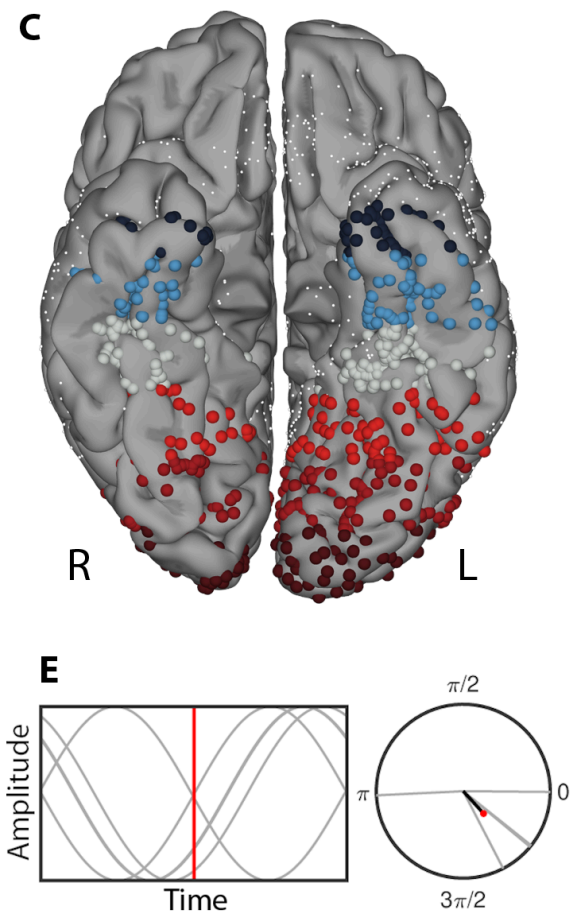

B

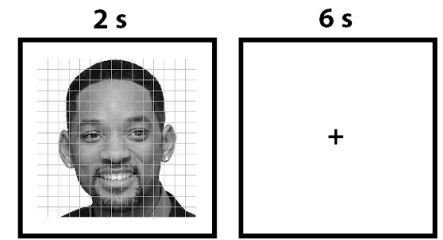

D

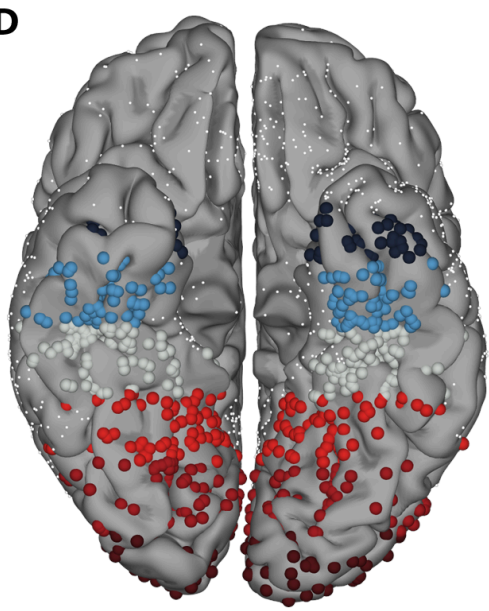

$\mathbf{F}$

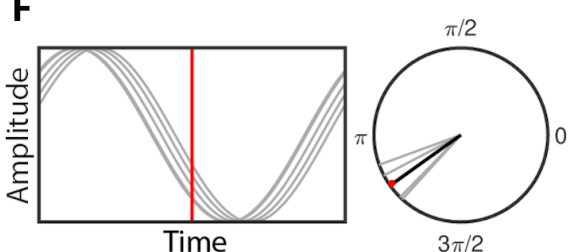

54 Figure 1: Tasks, Patients and ITC Analysis. (A,B) Schematic representation of the words (A) and faces

55 (B) tasks. (C,D) Individual electrode locations, within the ventral ROI, for patients reading words (C;

561,059 electrodes, 40 patients) and recognizing faces ( $D ; 1,246$ electrodes, 57 patients) plotted on a

57 standardized N27 brain. Electrode colors represent the relative locations with individual electrodes clustered into $20 \mathrm{~mm}$ bins along the y-axis. Smaller white spheres represent electrodes outside the ventral ROI. (E,F) Schematic representation with artificial data to illustrate low $(E ; I T C=0.25)$ and high $(F ;$ ITC $=0.95)$ phase alignment along with a representation of the vector mean. 


\section{Phase Modulation induced by Words and Faces}

63 Data from two representative patients, with broad coverage of ventral occipitotemporal cortex, are

64 shown in Figure 2. In both instances, increases in inter-trial phase coherence were seen in electrodes

65 from occipital pole to mid-fusiform cortex, for both tasks. Within each patient we also observed

66 highly comparable patterns of ITC across both tasks. As expected, during the period preceding the

67 onset of visual stimulation, the trial-by-trial distribution of phases at any given time was reasonably

68 uniform. However, following presentation of a visual stimulus, they tended to a coherent center

69 frequency (Figure $2 C, D)$. This phase coherence persisted for approximately 2 cycles ( $4 \pi$ rads, $300 \mathrm{~ms}$ )

70 (Figure $2 \mathrm{~F}, \mathrm{H}$ ). The temporal progression of the coherent center frequency was highly similar across

71 both tasks. 


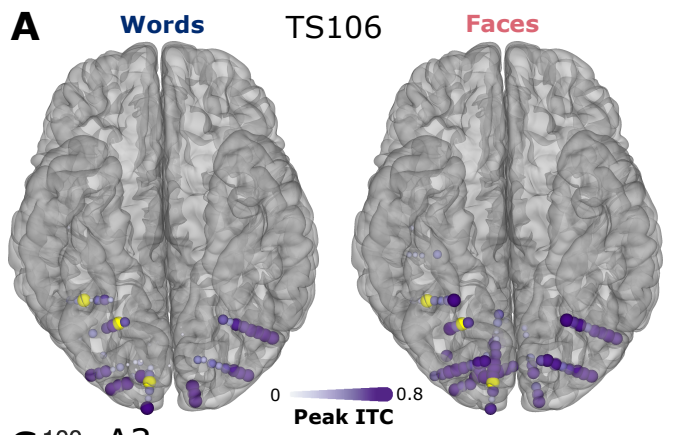

$\mathbf{C}^{100}[\mathrm{~A} 3$
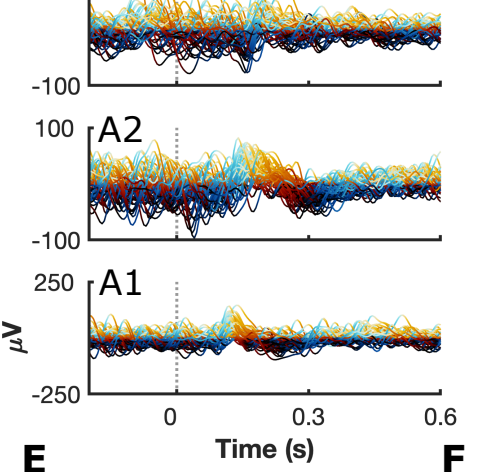

$\mathbf{E}$

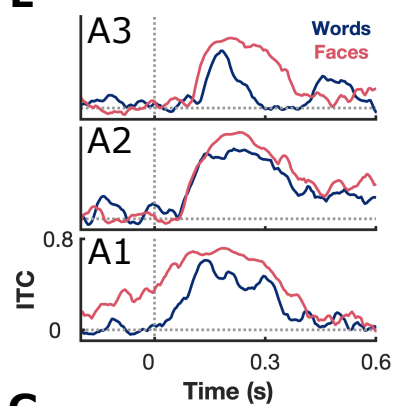

G

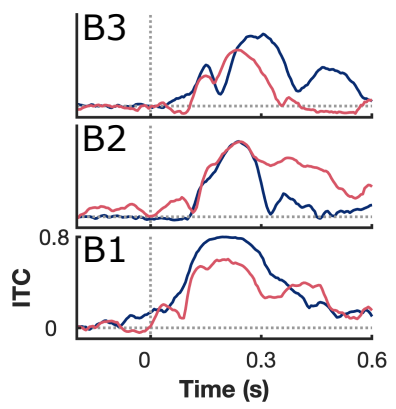

$\mathbf{F}$

H

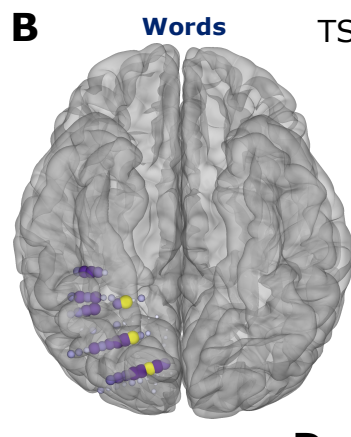

D
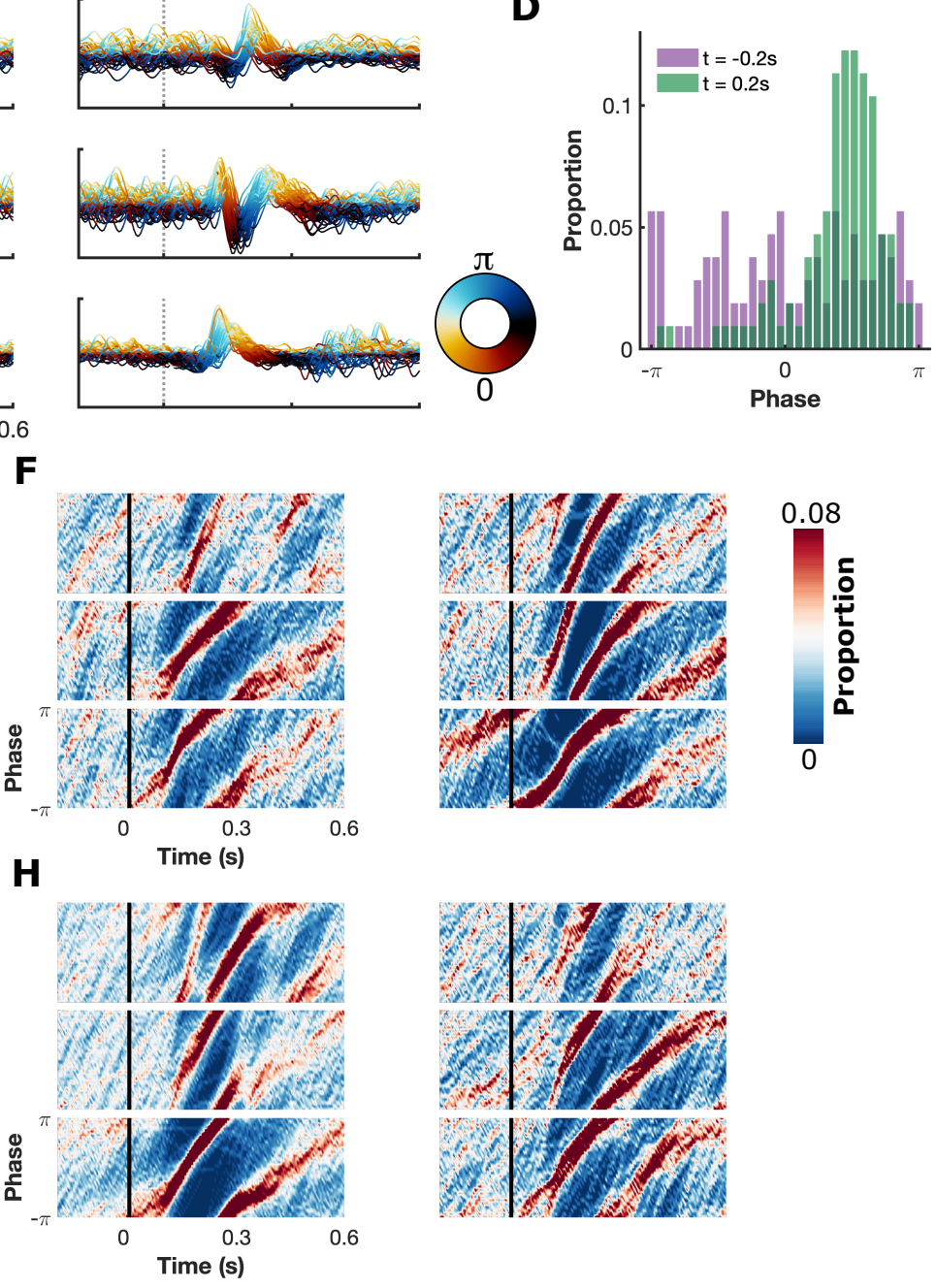

Figure 2: Spatial Propagation of Phase Coherence in Exemplar Patients. (A,B) ITC distributions, in

74 native patient space, for TS106 (A) and TS093 (B). Results are shown for words (left) and faces (right). (C) Broadband low frequency $(2-30 \mathrm{~Hz}$ ) signals of 60 trials from TS106 from the words (left) and faces (right) tasks. Traces colored based on instantaneous generalized phase. (D) Phase distribution for a

77 representative electrode at two time points during the faces task. (E-H) ITC $(E, G)$ and phase 78 distributions $(\mathrm{F}, \mathrm{H})$, with words (left) and faces (right) shown for three electrodes each from TS106 $79(\mathrm{E}, \mathrm{F})$ and TSO97 $(\mathrm{G}, \mathrm{H})$. Electrodes are highlighted in yellow in the brain plots and electrode numbers 80 increase from posterior to anterior. 
81 To evaluate the consistency of these effects across the population ( $n=82$ patients), we represented

82 ITC amplitudes on a group normalized cortical surface from occipital pole to anterior fusiform cortex

83 (Figure 3A,B). High ITC (>0.5) values were noted in associative cortex as far anterior as mid-fusiform.

84 Stimulus presentation resulted in two distinct peaks of the ITC measure - a higher one around stimulus onset and a smaller one at offset. Differences in offset time between the two tasks, based on experimental design, confirmed that this was an offset response (Figure 3D,E). Results calculated using the raw signal $(0.3-100 \mathrm{~Hz})$ showed highly correlated peak ITC with the broadband low frequency $(2-30 \mathrm{~Hz})$ signal (Spearman correlation; words, $r(1,059)=0.86, p<0.001$; faces, $r(1,246)=$ $0.82, \mathrm{p}<0.001$; Supplementary Figure $1 \mathrm{E}, \mathrm{F})$ but with the raw signal displaying a longer duration of significant ITC (Supplementary Figure 1G,H).

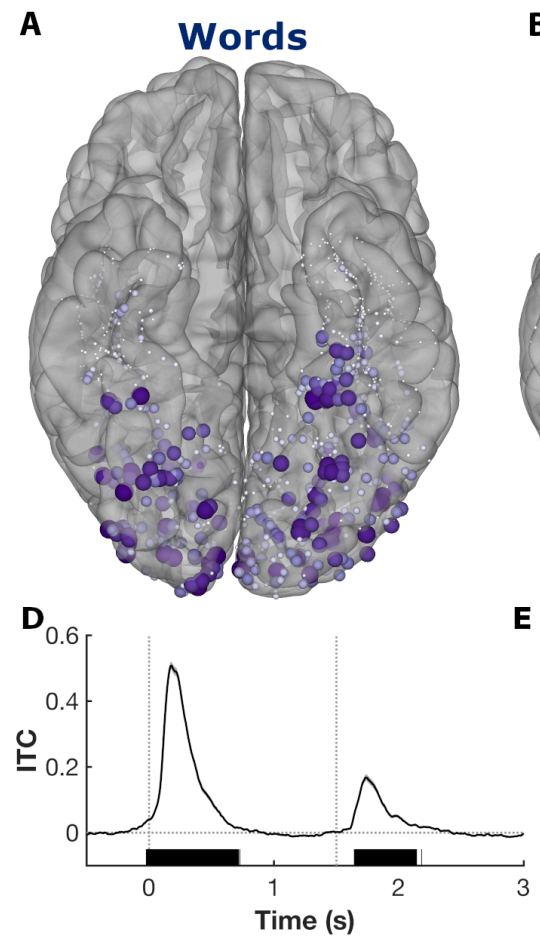

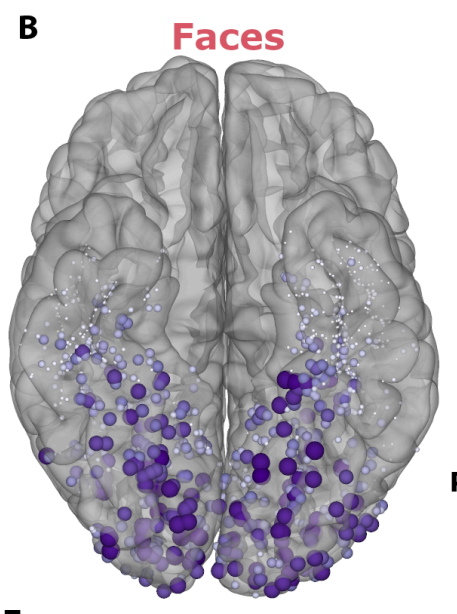

$\mathbf{E}$

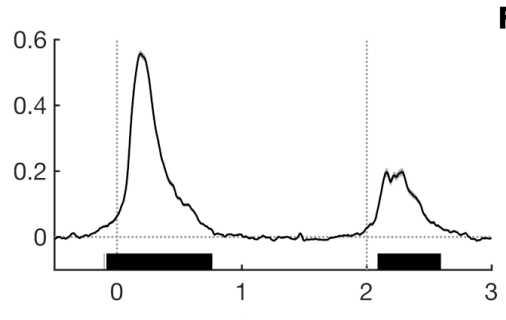

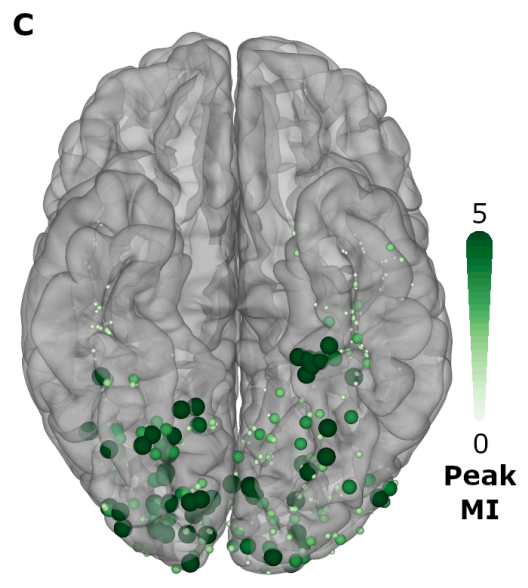

$\mathbf{F}$

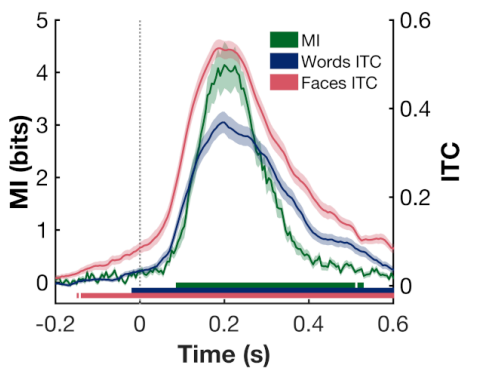

Figure 3: Population Phase Coherence. (A,B) Peak ITC at individual electrodes for words (A; 1,059 electrodes) and faces ( $B ; 1,246$ electrodes) tasks represented on a standardized surface. (C) Peak mutual information (MI) between the phase distributions in response to word or face presentation in patients who performed both tasks (354 electrodes, 15 patients). (D,E) Time course of ITC (mean

$96 \pm$ SE) at electrodes with the top $20 \%$ peak amplitude ITC responses to words (D) and faces (E). Vertical 97 lines denote the onset and offset times of each stimulus. Colored bars represent regions of significant 98 increase over baseline ( $q<0.001$ ). (F) MI and ITC for the electrodes with the top $20 \%$ highest peak MI.

99 See also Supplementary Figures 1, 2. 
100 The results thus far demonstrate that within each task there is phase coherence between trials and

101 the magnitude and duration of this coherence is reasonably conserved across different tasks. But, 102 how consistent is this pattern of evoked phase across tasks? To quantify this, we measured mutual 103 information (MI) in patients who performed both tasks $(n=15)$. We observed an increase in MI 104 following the visual onsets, representing an increase in the similarity of phase distributions between 105 tasks. This inter-task coherence peaked at $\sim 200 \mathrm{~ms}$ following visual onset, highly concordant with the 106 within-trial ITC (Figure 3F). 9 out of the 15 patients had at least one electrode peaking at $>5$ bits 107 (48/354 electrodes; Supplementary Figure 2A). Peak MI correlated well with peak ITC, measured 108 across both tasks (Spearman correlation, $r(354)=0.76, p<0.001$; Supplementary Figure $2 \mathrm{C}$ ).

109 Next, we sought to characterize the spatiotemporal properties of this response, by dividing the 110 ventral cortical surface into $20 \mathrm{~mm}$ bins along the $y$ axis of the normalized surface in Talairach space, 111 and used this to compare grouped ITC profiles from each bin (Figure 4). We noted a pronounced 112 posterior-to-anterior gradient of both amplitude and onset latency of ITC with the largest responses 113 in early visual cortex and progressive diminution anteriorly in both hemispheres, for both tasks. A 4D, 114 cortical surface-based representation of the propagation of this phase coherence across the cortical 115 surface revealed a posterior-to-anterior wave, highly conserved across both tasks (Movie 1).

117 Movie 1: Spread of Phase Coherence across the Cortical Surface. Population-level, surface-based 118 representation of ITC in response to words (left) and faces (right).

To quantify this posterior-to-anterior latency gradient, we calculated the time point at which the first 121 derivative of the ITC first significantly deviated from baseline at each electrode (Figure 4E). In both 122 tasks there was a clear gradient from calcarine to anterior fusiform. Onset latency showed a 123 significant association with position in the anteroposterior axis for both words $(t(781)=12.5, \beta=1.0$ $\left.124 \pm 0.08, p<0.001, r^{2}=0.30\right)$ and faces $\left(t(779)=9.7, \beta=1.3 \pm 0.13, p<0.001, r^{2}=0.21\right)$ tasks. This 125 reflects a posterior-to-anterior progression of the timing of ITC across the cortical surface from early 126 sensory cortex to associative regions and a consistent propagation rate of approximately 0.8-1 m/s 127 in Euclidean space.

128 To further investigate the spatiotemporal consistency of this effect between tasks, we analyzed just 129 the subset of patients who performed both tasks $(n=15)$. There was no significant difference in ITC 
onset latency between the two tasks $(\mathrm{t}(462)=-1.6, \beta=-0.03 \pm 0.02, \mathrm{p}=0.12)$ or any significant effect of task on the propagation rate $(t(462)=-1.3, \beta=-0.35 \pm 0.27, p=0.20)$.
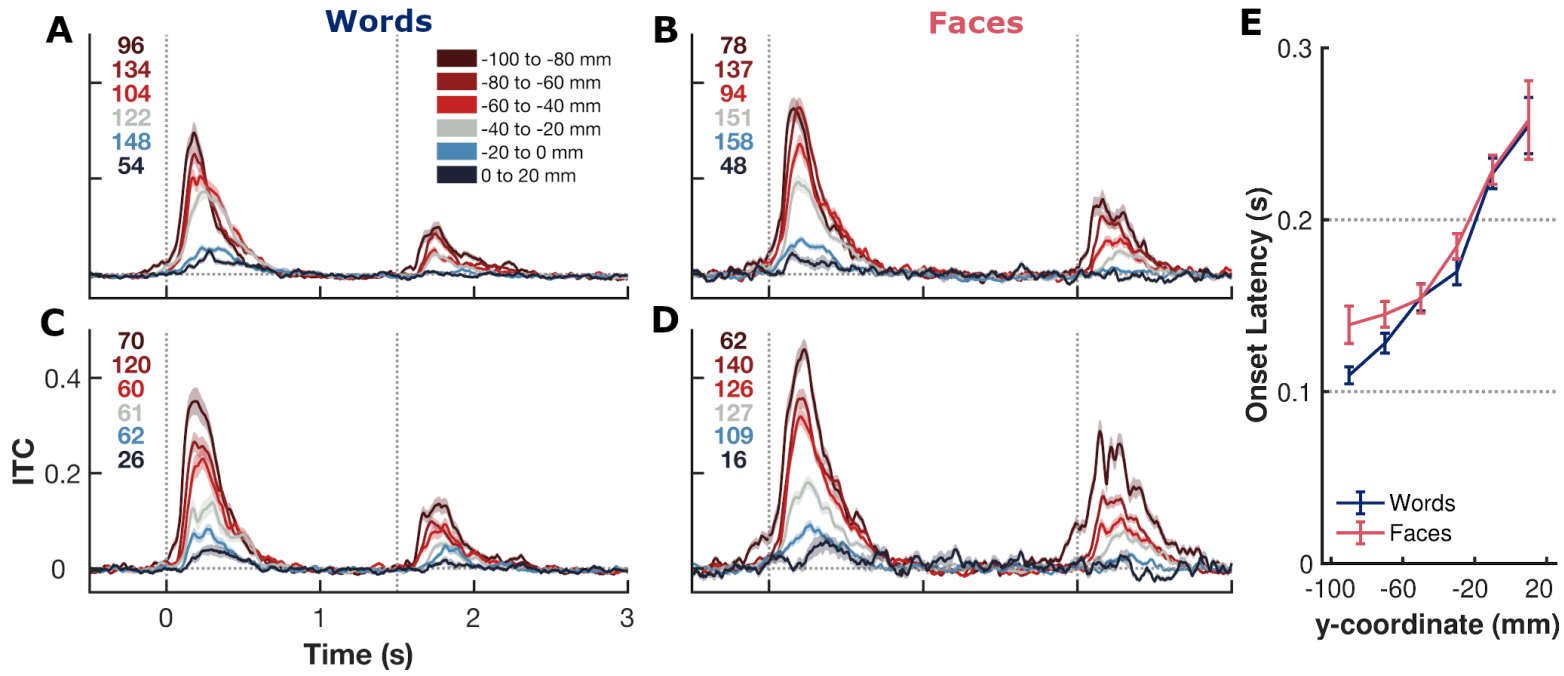

133 Figure 4: ITC - Positional Analysis. (A-D) ITC in response to words (A,C) or faces (B,D) as a function of 134 anteroposterior position in the left $(A, B)$ and right $(C, D)$ hemisphere. Presented as mean $\pm \mathrm{SE}$. 135 Number of electrodes in each ROI shown. Vertical lines denote stimulus onset and offset times. (E) 136 ITC demonstrates a posterior-to-anterior gradient of first onset latency (ITC first-derivative >3.5SD 137 deviation from baseline).

139 To further characterize this posterior-to-anterior spread and to identify the degree of propagation of 140 phase information across the cortical surface, we calculated the lagged phase locking value (PLV) 141 between pairs of electrodes. By lagging the time courses of phase progression between pairs of 142 electrodes we sought to determine to what degree the future phase of anterior sites could be 143 predicted by the current phase of posterior sites (Figure 5A). This analysis demonstrated a strong bias 144 toward a posterior-to-anterior spread of phase information, with the phase of anterior sites being 145 driven by more posterior regions (Figure 5B, Supplementary Figure 3). 


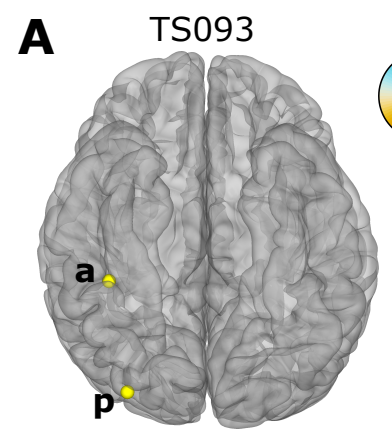

B
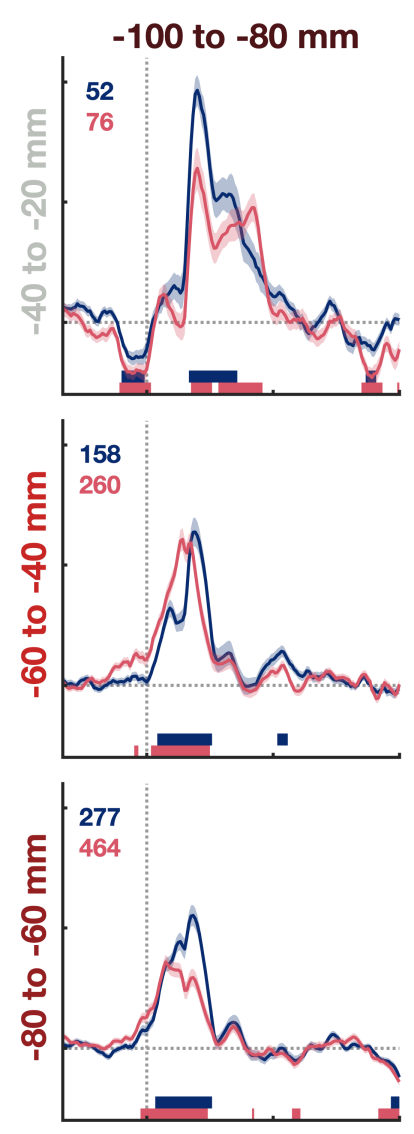

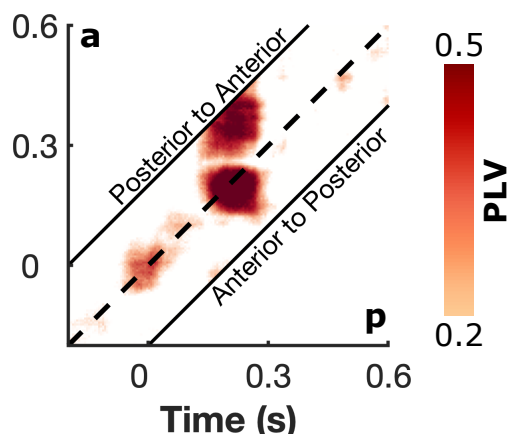

Time (s)
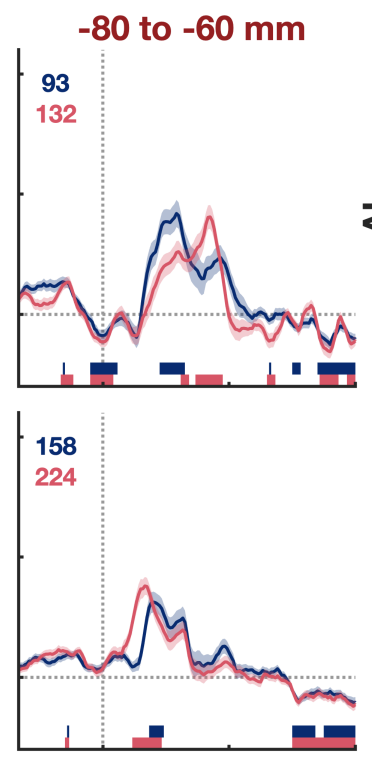

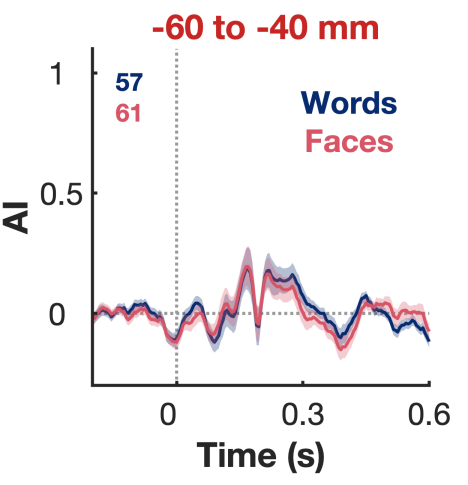

147 Figure 5: Propagation of Phase. (A) Exemplar electrode pair demonstrating the lagged phase locking 148 value (PLV) analysis. PLV was calculated between the instantaneous phase of the posterior electrode 149 (p) and the time lagged phase of the anterior electrode (a; -200 to $200 \mathrm{~ms}$ ). Greater PLV above the 150 center diagonal (dashed line) represents a greater ability to predict future phase of the anterior 151 electrode based on the current phase of the posterior electrode. (B) Calculated asymmetry index (AI) 152 of PLV above and below the center diagonal for high ITC (>0.5) electrode pairs across ROIs. Al $>0$ 153 represents a bias toward posterior-to-anterior phase propagation. Colored bars represent regions of 154 significant difference from baseline $(q<0.001)$. Number of electrode pairs in each ROI pair shown. See also Supplementary Figure 3. 


\section{Discussion}

157 We have identified a visually-induced, macroscale, spatiotemporal propagation of phase modulation

158 from posterior-to-anterior in human ventral occipitotemporal cortex. Strong phase modulation is 159 seen almost ubiquitously across ventral occipitotemporal cortex in both hemispheres; from occipital 160 pole to anterior fusiform. Following a visual event each region within the visual pathway displays a 161 predictable, stereotyped phase progression. These findings were highly repeatable across a diverse 162 range of visual stimuli with varying task demands and attentional loads and in only partially overlapping patient populations. We observed an approximate Euclidean propagation velocity of 0.8 -

$1641.0 \mathrm{~m} / \mathrm{s}$. This is comparable to the conduction velocities of unmyelinated horizontal projection axons 165 in macaques (0.1-0.6 m/s) (Davis et al., 2020) and is much slower than would be expected from 166 monosynaptic white matter transmission (>15 m/s) (Ferraina et al., 2002). This further implies that 167 this phase propagation likely occurs via sequential cortico-cortical connections (Muller et al., 2014).

168 Previous work has shown the organization of spontaneous oscillations as large scale travelling waves 169 (Bahramisharif et al., 2013; Muller et al., 2016; Zhang et al., 2018) and small scale travelling waves 170 within early visual cortex (Muller et al., 2014; Sato et al., 2012). We expand on this work, 171 characterizing a visually-evoked, macroscale phase propagation across the entire ventral visual 172 pathway that likely indexes feedforward communication (Bastos et al., 2015), long-range cortico173 cortical connections (Muller et al., 2014) and integration of information over broad cortical areas 174 (Sato et al., 2012; Zhang et al., 2018).

175 A phase reset following visual input has been demonstrated in human (Mormann et al., 2005; Rizzuto 176 et al., 2003; Tesche and Karhu, 2000) and macaque (Jutras et al., 2013) hippocampi. It is possible that 177 this is also initiated as part of this sequence of ventral phase modulations. During visual exploration 178 this hippocampal phase reset consistently occurs following saccadic eye movements (Jutras et al., 179 2013) suggesting that ventral phase modulation may occur following each saccade during naturalistic 180 viewing.

181 Extracranial recordings of study phase modulation are an aggregate of many sources, limiting insight 182 into underlying generators. Further, the relative inaccessibility of electrical fields generated by the 183 ventral temporal surface (Goldenholz et al., 2009) has limited studies of visual phase modulation to 184 early visual cortex. This has precluded insight into the spatiotemporal topography of visually-induced 185 phase modulation in the human ventral visual pathway. Our results are supportive of the phase reset 
model of event related potential (ERP) generation (Klimesch et al., 2007; Makeig et al., 2002; Sauseng et al., 2007; Yeung et al., 2004). According to the phase reset model, the reset of instantaneous phase of ongoing oscillations, tending toward a fixed value upon presentation of a stimulus, results in the ERP by creating a coherent superposition of phase components (lemi et al., 2019). Simulations of the underlying generators of the ERP suggest that early components ( $<200 \mathrm{~ms})$, corresponding to the time course of ITC seen here, primarily represent feed-forward influences, with later ERP components being influenced by top-down modulation (Garrido et al., 2007).

\section{Conclusion}

We demonstrate the existence of visually-induced macroscale posterior-to-anterior propagation of phase modulation across the human ventral occipitotemporal cortex. We propose this represents a feed-forward spread of information via horizontal cortico-cortical connections throughout the ventral visual pathway.

\section{Materials and Methods}

Participants: 82 patients (44 male, 18-60 years, 16 left-handed) undergoing implantation with intracranial electrodes for seizure localization of pharmaco-resistant epilepsy participated after giving written informed consent. Participants with significant additional neurological history (e.g. previous resections, MR imaging abnormalities such as malformations or hypoplasia) were excluded. All experimental procedures were reviewed and approved by the Committee for the Protection of Human Subjects (CPHS) of the University of Texas Health Science Center at Houston as Protocol Number HSC-MS-06-0385.

Electrode Implantation and Data Recording: Data were acquired using either subdural grid electrodes (SDEs; 14 patients) or stereotactically placed depth electrodes (sEEGs; 68 patients). SDEs were subdural platinum-iridium electrodes embedded in a silicone elastomer sheet (PMT Corporation; tophat design; 3mm diameter cortical contact), surgically implanted via a craniotomy following previously described methods (Pieters et al., 2013; Tandon, 2012; Tong et al., 2020). sEEGs were implanted using a Robotic Surgical Assistant (ROSA; Medtech, Montpellier, France) (Rollo et al., 2020; Tandon et al., 2019). Each sEEG probe (PMT corporation, Chanhassen, Minnesota) is $0.8 \mathrm{~mm}$ in 
215 diameter, with 8-16 electrode contacts, each contact being a $2 \mathrm{~mm}$ long platinum-iridium cylinder 216 separated from the adjacent contact by $1.5-2.43 \mathrm{~mm}$. Each patient had 12-20 such probes

217 implanted. Following surgical implantation, electrodes were localized by co-registration of pre218 operative anatomical 3T MRI and post-operative CT scans in AFNI (Cox, 1996). Electrode positions 219 were projected onto a cortical surface model generated in FreeSurfer (Dale et al., 1999), and 220 displayed on the cortical surface model for visualization (Pieters et al., 2013). Intracranial data were 221 collected during research experiments starting on the first day after electrode implantation for sEEGs 222 and two days after implantation for SDEs. Data were digitized at $2 \mathrm{kHz}$ using the NeuroPort recording 223 system (Blackrock Microsystems, Salt Lake City, Utah), imported into Matlab, and visually inspected 224 for line noise, artifacts and epileptic activity. Of a total of 11,164 electrodes, 3,919 electrodes with 225 obvious line noise or localized in proximity to sites of seizure onset were excluded. Each electrode 226 was re-referenced to the common average of the remaining channels. Trials contaminated by inter227 ictal epileptic spikes were discarded.

228 Signal Analysis: An ROI encompassing the entire occipital lobe and the ventral temporal surface, but 229 excluding parahippocampal and entorhinal regions, was then applied, restricting us to 1,929 230 electrodes which form the basis of this work. These electrodes were grouped into sub-ROIs within 20 $231 \mathrm{~mm}$ intervals along the $\mathrm{y}$-axis of Talairach space, from $-100 \mathrm{~mm}$ to $20 \mathrm{~mm}$ (Figure 1D-F).

232 Raw data was notch filtered to remove line noise (zero-phase 2nd order Butterworth band-stop 233 filters). Phase information was extracted from the down-sampled (200 Hz) and wide band-pass 234 filtered data (2 - $30 \mathrm{~Hz}$; zero-phase 8th order Butterworth band-pass filter) using the 'generalized 235 phase' method (Davis et al., 2020) with a single-sided Fourier transform approach (Marple, 1999). 236 This method captures the phase of the predominant fluctuations in the wideband signal and 237 minimizes filter-related distortion of the waveform.

238 Inter-trial Phase Coherence (ITC) was calculated as the absolute vector length of the mean of unit 239 vectors with the instantaneous phase of stimulus-aligned trials (Figure 1G,H). Phase locking value 240 (PLV) was calculated identically to ITC but instead using phase difference between two electrodes. 241 ITC and PLV were computed as the median value of 50 iterations of a random 60 trials. ITC and PLV 242 were baselined $-1,000$ to -100 ms before each stimulus. PLV was calculated between pairs of 243 electrodes in separate ROIs, within patient, within hemisphere. PLV statistics were performed using 244 a bootstrapped null distribution, randomly re-pairing trials between electrodes, using 5,000 repetitions. Phase probability distributions were quantified, using 30 equally spaced bins between - 
$\pi$ and $\pi$, and mutual information (MI) was calculated based on these distributions at each time point, baselined $-1,000$ to -100 ms before each stimulus. Asymmetry index (AI) was calculated based on the

248 lagged PLV results at each time point as $A l=\log _{2}\left(P L V_{p a} / P L V_{a p}\right)$, where PLV 249 between electrode $p$ at time $t$ and electrode $a$ between time $t$ and time $t+200 \mathrm{~ms}$.

250 Experimental Design and Statistical Analysis: Patients read single words ( $\mathrm{n}=40$ patients) or identified 251 celebrity faces $\left(n=57\right.$ patients). Stimuli were presented on a $2,880 \times 1,800,15.4^{\prime \prime}$ LCD screen 252 positioned at eye-level, 2-3' from the patient. Additional details of each experiment are below:

Word Reading: Participants read aloud monosyllabic words (e.g. dream) and pseudowords (e.g. meech) presented in lower-case Arial font, 150 pixels high $\left(\sim 2.2^{\circ}\right.$ visual angle) (Figure $\left.1 \mathrm{~A}\right)$ (Woolnough et al., 2021a). Stimuli were presented using Psychophysics Toolbox (Kleiner et al., 2007) run in Matlab. Each stimulus was displayed for 1,500 ms with an inter-stimulus interval of Face Recognition: Participants were presented with greyscale photos of famous faces and asked to name them aloud (Figure 1B) (Kadipasaoglu et al., 2017; Woolnough et al., 2020). Stimuli were matched for luminance and contrast and presented, using Python 2.7, at a size of $500 \times 500$ pixels

Linear regressions were performed using robust regression models (Matlab function fit/m using 'RobustOpts'; iteratively reweighted least squares with bisquare weighting) (Welsch, 1977), to minimize the effect of outliers and to make fewer assumptions than traditional least squares linear regression models. To calculate onset latencies, we determined the first time point per electrode, within $600 \mathrm{~ms}$ of stimulus onset, where the first derivative of the ITC exceeded 3.5 standard deviations $(\sim p<0.001)$ from baseline. We then used a regression model, predicting onset latency based on position in the $y$ (anteroposterior) axis, derived from the surface-based co-registration. All

272 temporal analyses were corrected for multiple comparisons using a Benjamini-Hochberg false273 detection rate (FDR) threshold of $\mathrm{q}<0.001$. 


\section{Acknowledgements}

275 We express our gratitude to all the patients who participated in this study; the neurologists at the

276 Texas Comprehensive Epilepsy Program who participated in the care of these patients; and the 277 nurses and technicians in the Epilepsy Monitoring Unit at Memorial Hermann Hospital who helped 278 make this research possible. This work was supported by the National Institute for Deafness and 279 other Communication Disorders DC014589 and National Institute of Neurological Disorders and 280 Stroke NS098981.

\section{Author Contributions}

282 Conceptualization: OW, NT; Methodology: OW, NT; Data curation: OW, PSR, ZR; Software: OW; 283 Formal Analysis and Visualization: OW; Writing - Original Draft: OW; Writing - Review and Editing: 284 OW, KJF, NT; Funding Acquisition: NT; Neurosurgical Procedures: NT.

\section{Declaration of Interests}

286 The authors declare no competing interests 


\section{References}

288 Alamia A, VanRullen R. 2019. Alpha oscillations and traveling waves: Signatures of predictive coding? PLoS Biol 17. doi:10.1371/journal.pbio.3000487

Arnal LH, Giraud AL. 2012. Cortical oscillations and sensory predictions. Trends Cogn Sci 16:390398. doi:10.1016/j.tics.2012.05.003

Bahramisharif A, van Gerven MAJ, Aarnoutse EJ, Mercier MR, Schwartz TH, Foxe JJ, Ramsey NF, Jensen O. 2013. Propagating neocortical gamma bursts are coordinated by traveling alpha waves. J Neurosci 33:18849-18854. doi:10.1523/JNEUROSCI.2455-13.2013

Bonnefond M, Jensen O. 2012. Alpha oscillations serve to protect working memory maintenance against anticipated distracters. Curr Biol 22:1969-1974. doi:10.1016/j.cub.2012.08.029

Cox RW. 1996. AFNI: Software for Analysis and Visualization of Functional Magnetic Resonance Neuroimages. Comput Biomed Res 29:162-173. doi:10.1006/cbmr.1996.0014

Dale AM, Fischl B, Sereno MI. 1999. Cortical Surface-Based Analysis: I. Segmentation and Surface Reconstruction. Neuroimage 9:179-194. doi:10.1006/nimg.1998.0395

Davis Z, Muller L, Trujillo J-M, Sejnowski T, Reynolds J. 2020. Spontaneous Traveling Cortical Waves Gate Perception in Awake Behaving Primates. Nature. doi:10.1038/s41586-020-2802-y

Ferraina S, Paré M, Wurtz RH. 2002. Comparison of cortico-cortical and cortico-collicular signals for the generation of saccadic eye movements. J Neurophysiol 87:845-858. doi:10.1152/jn.00317.2001

Forseth KJ, Hickok G, Rollo PS, Tandon N. 2020. Language prediction mechanisms in human auditory cortex. Nat Commun 11:5240. doi:10.1038/s41467-020-19010-6 
315 Forseth KJ, Kadipasaoglu CM, Conner CR, Hickok G, Knight RT, Tandon N. 2018. A lexical semantic hub for heteromodal naming in middle fusiform gyrus. Brain 141:2112-2126. doi:10.1093/brain/awy120

Garrido MI, Kilner JM, Kiebel SJ, Friston KJ. 2007. Evoked brain responses are generated by

Ghuman AS, Brunet NM, Li Y, Konecky RO, Pyles JA, Walls SA, Destefino V, Wang W, Richardson RM. 2014. Dynamic encoding of face information in the human fusiform gyrus. Nat Commun

Goldenholz DM, Ahlfors SP, Hämäläinen MS, Sharon D, Ishitobi M, Vaina LM, Stufflebeam SM. 2009. Mapping the signal-to-noise-ratios of cortical sources in magnetoencephalography and

Halgren M, Ulbert I, Bastuji H, Fabó D, Eross L, Rey M, Devinsky O, Doyle WK, Mak-McCully R, Halgren E, Wittner L, Chauvel P, Heit G, Eskandar E, Mandell A, Cash SS. 2019. The generation and propagation of the human alpha rhythm. Proc Natl Acad Sci 116:23772-23782. doi:10.1073/pnas.1913092116

Hirshorn EA, Li Y, Ward MJ, Richardson RM, Fiez JA, Ghuman AS. 2016. Decoding and disrupting left midfusiform gyrus activity during word reading. Proc Natl Acad Sci 113:8162-8167. doi:10.1073/pnas.1604126113

Howard MF, Poeppel D. 2012. The neuromagnetic response to spoken sentences: Co-modulation of theta band amplitude and phase. Neuroimage 60:2118-2127. doi:10.1016/j.neuroimage.2012.02.028

342 Kadipasaoglu CM, Conner CR, Baboyan VG, Rollo M, Pieters TA, Tandon N. 2017. Network dynamics of human face perception. PLoS One 12. doi:10.1371/journal.pone.0188834 
344 Kadipasaoglu CM, Conner CR, Whaley ML, Baboyan VG, Tandon N. 2016. Category-selectivity in 345 human visual cortex follows cortical topology: A grouped icEEG study. PLoS One 11. doi:10.1371/journal.pone.0157109

Kleiner M, Brainard D, Pelli D. 2007. What's new in Psychtoolbox-3? Perception 36. doi:10.1068/v070821

Klimesch W, Sauseng P, Hanslmayr S, Gruber W, Freunberger R. 2007. Event-related phase reorganization may explain evoked neural dynamics. Neurosci Biobehav Rev 31:1003-1016. doi:10.1016/j.neubiorev.2007.03.005

Lakatos P, Karmos G, Mehta AD, Ulbert I, Schroeder CE. 2008. Entrainment of neuronal oscillations

Lakatos P, Shah AS, Knuth KH, Ulbert I, Karmos G, Schroeder CE. 2005. An oscillatory hierarchy controlling neuronal excitability and stimulus processing in the auditory cortex. J Neurophysiol

Lamme VAF, Roelfsema PR. 2000. The distinct modes of vision offered by feedforward and 94:1904-1911. doi:10.1152/jn.00263.2005 recurrent processing. Trends Neurosci 23:571-579. doi:10.1016/S0166-2236(00)01657-X

Lozano-Soldevilla D, VanRullen R. 2019. The Hidden Spatial Dimension of Alpha: 10-Hz Perceptual

Luo H, Liu Z, Poeppel D. 2010. Auditory cortex tracks both auditory and visual stimulus dynamics using low-frequency neuronal phase modulation. PLoS Biol 8:25-26. doi:10.1371/journal.pbio.1000445 Echoes Propagate as Periodic Traveling Waves in the Human Brain. Cell Rep 26:374-380. doi:10.1016/j.celrep.2018.12.058 
Mishkin M, Ungerleider LG, Macko KA. 1983. Object vision and spatial vision: two cortical pathways.

Morán MA, Mufson EJ, Mesulam M -M. 1987. Neural inputs into the temporopolar cortex of the rhesus monkey. J Comp Neurol 256:88-103. doi:10.1002/cne.902560108

Mormann F, Fell J, Axmacher N, Weber B, Lehnertz K, Elger CE, Fernández G. 2005.

Muller L, Chavane F, Reynolds J, Sejnowski TJ. 2018. Cortical travelling waves: Mechanisms and computational principles. Nat Rev Neurosci 19:255-268. doi:10.1038/nrn.2018.20

Muller L, Reynaud A, Chavane F, Destexhe A. 2014. The stimulus-evoked population response in

Rizzuto DS, Madsen JR, Bromfield EB, Schulze-Bonhage A, Seelig D, Aschenbrenner-Scheibe R, Kahana MJ. 2003. Reset of human neocortical oscillations during a working memory task. Proc Natl Acad Sci 100:7931-6. doi:10.1073/pnas.0732061100

Rollo PS, Rollo MJ, Zhu P, Woolnough O, Tandon N. 2020. Oblique trajectory angles in robotic stereo-electroencephalography. J Neurosurg. doi:10.3171/2020.5.JNS20975 
discussion. Neuroscience 146:1435-1444. doi:10.1016/j.neuroscience.2007.03.014

403

Tandon N. 2012. Mapping of human language In: Yoshor D, Mizrahi E, editors. Clinical Brain Mapping. McGraw Hill Education. pp. 203-218.

Tandon N, Tong BA, Friedman ER, Johnson JA, Von Allmen G, Thomas MS, Hope OA, Kalamangalam GP, Slater JD, Thompson SA. 2019. Analysis of Morbidity and Outcomes Associated With Use of Subdural Grids vs Stereoelectroencephalography in Patients With Intractable Epilepsy. JAMA Neurol 76:672-681. doi:10.1001/jamaneurol.2019.0098

Tang H, Buia C, Madhavan R, Crone NE, Madsen JR, Anderson WS, Kreiman G. 2014. Spatiotemporal Dynamics Underlying Object Completion in Human Ventral Visual Cortex. Neuron 83:736-748. doi:10.1016/j.neuron.2014.06.017

Tesche CD, Karhu J. 2000. Theta oscillations index human hippocampal activation during a working memory task. Proc Natl Acad Sci 97:919-924. doi:10.1073/pnas.97.2.919

Tong BA, Esquenazi Y, Johnson J, Zhu P, Tandon N. 2020. The Brain is Not Flat: Conformal Electrode Arrays Diminish Complications of Subdural Electrode Implantation, A Series of 117 Cases. World Neurosurg 144:e734-e742. doi:10.1016/j.wneu.2020.09.063

Welsch RE. 1977. Robust regression using iteratively reweighted least-squares. Commun Stat Theory Methods 6:813-827. doi:10.1080/03610927708827533

Woolnough O, Donos C, Curtis A, Rollo PS, Roccaforte ZJ, Tandon N. 2021a. A Spatiotemporal Map of Reading Aloud. bioRxiv. doi:10.1101/2021.05.23.445307

Woolnough O, Donos C, Rollo PS, Forseth KJ, Lakretz Y, Crone NE, Fischer-Baum S, Dehaene S, Tandon N. 2021b. Spatiotemporal dynamics of orthographic and lexical processing in the ventral visual pathway. Nat Hum Behav 5:389-398. doi:10.1038/s41562-020-00982-w

Woolnough O, Rollo PS, Forseth KJ, Kadipasaoglu CM, Ekstrom AD, Tandon N. 2020. Category Selectivity for Face and Scene Recognition in Human Medial Parietal Cortex. Curr Biol 30:27072715. doi:10.1016/j.cub.2020.05.018

Yeung N, Bogacz R, Holroyd CB, Cohen JD. 2004. Detection of synchronized oscillations in the electroencephalogram: An evaluation of methods. Psychophysiology 41:822-832. doi:10.1111/j.1469-8986.2004.00239.x

Zhang H, Watrous AJ, Patel A, Jacobs J. 2018. Theta and Alpha Oscillations Are Traveling Waves in 
bioRxiv preprint doi: https://doi.org/10.1101/2021.08.02.454804: this version posted August 4, 2021. The copyright holder for this preprint (which was not certified by peer review) is the author/funder, who has granted bioRxiv a license to display the preprint in perpetuity. It is made available under aCC-BY-NC-ND 4.0 International license. 
A
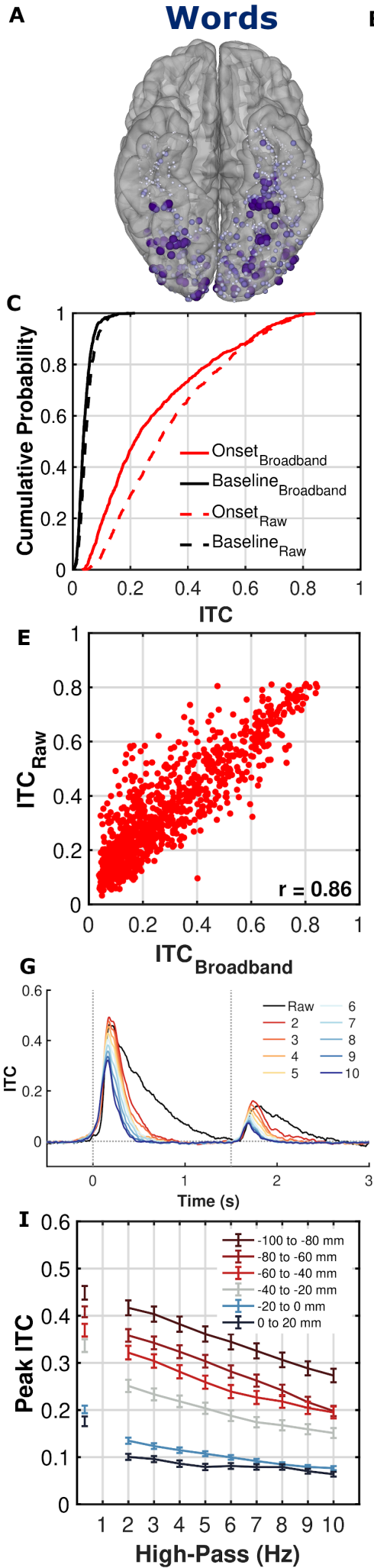

B
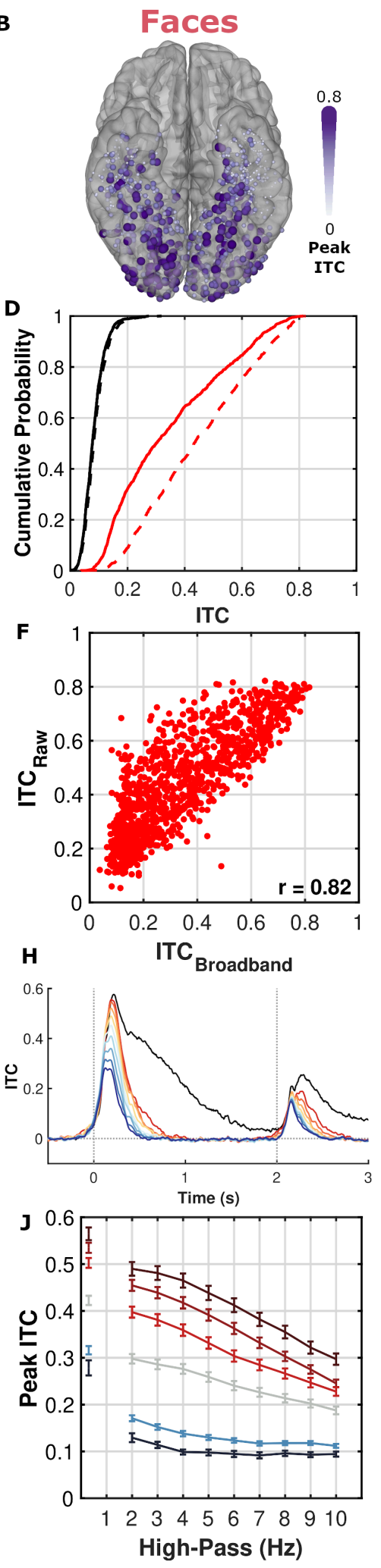

Supplementary Figure 1: Comparison of Broadband Low Frequency and Raw Signal Analyses. (A,B)

436 Spatial distribution of peak ITC for each electrode in the raw $(0.3-100 \mathrm{~Hz})$ signal. (C,D) Cumulative 437 probability distribution of raw and broadband ITC. (E,F) Relationship of raw and broadband ITC. 
$438(G, H)$ Temporal ITC profile with varying high pass filter parameters. Same electrodes as Figure 3D,E.

439 (I,J) Changes in peak ITC within each spatial ROI for the raw signal ( $0.3 \mathrm{~Hz}$ high pass) and varying

440 high pass filtered signals $(2-10 \mathrm{~Hz})$. Analyses shown for the words $(A, C, E, G, I)$ and faces $(B, D, F, H, J)$

441 tasks.
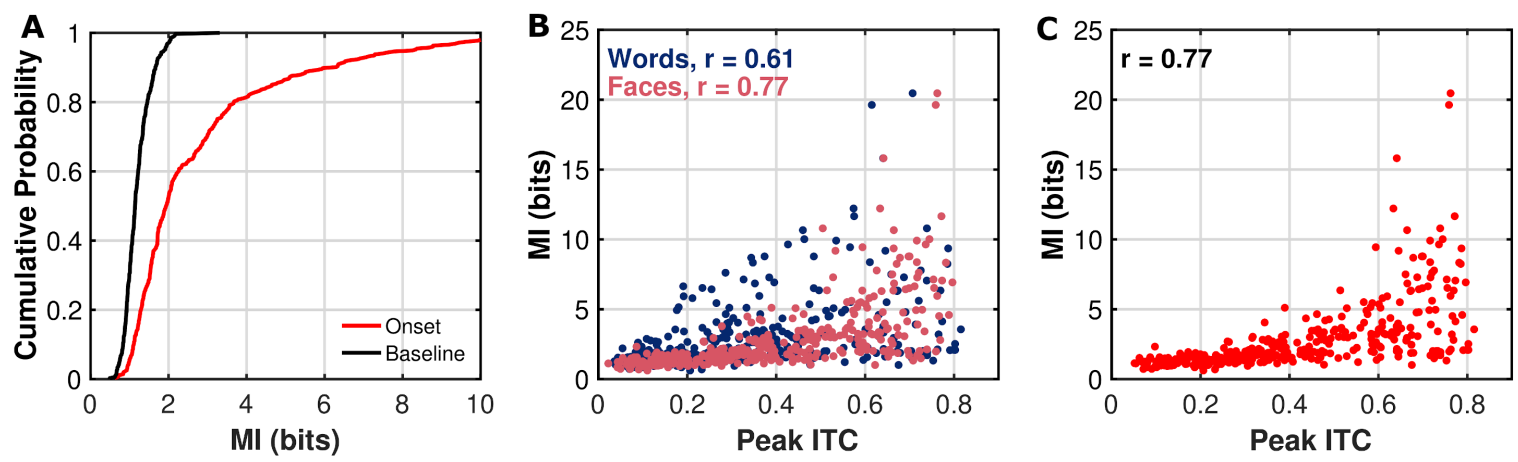

444 Supplementary Figure 2: Distribution of Mutual Information (MI). (A) Cumulative distribution

445 function of peak MI within the onset window (0 to $600 \mathrm{~ms}$; red) or during the baseline window (-

446500 to $-100 \mathrm{~ms}$; black). (B,C) Relationship between peak MI and (B) peak ITC in the words and faces

447 tasks or (C) peak ITC across both tasks, within individual electrodes. 
A
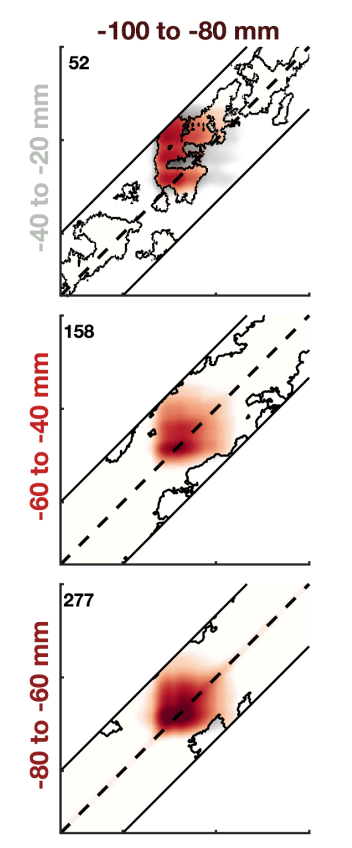

B
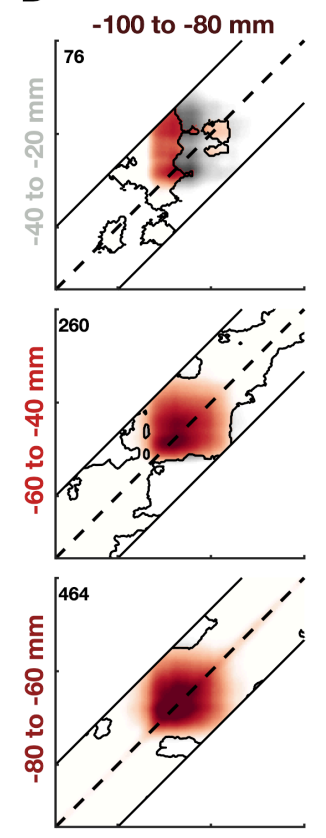

Words
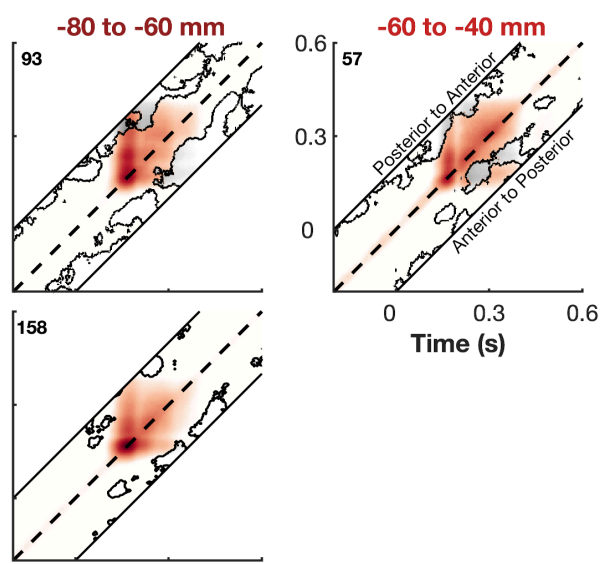

Faces
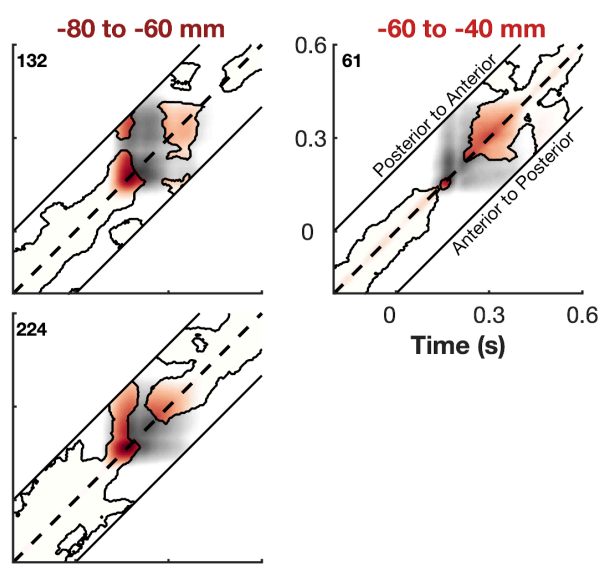

449 Supplementary Figure 3: Lagged Phase Locking Value. PLV was calculated between the

450 instantaneous phase of the posterior electrodes (columns) and the time lagged phase of the

451 anterior electrodes (rows; -200 to $200 \mathrm{~ms}$ ) for the words (A) and faces (B) tasks. Greater PLV above

452 the center diagonal (dashed line) represents a greater ability to predict future phase of the anterior

453 electrodes based on the current phase of the posterior electrodes. Areas of significant PLV (q<

454 0.05) above a trial shuffled null distribution are indicated by contours and non-significant areas are desaturated. Number of electrode pairs in each ROI pair shown. 